\title{
The ARIC predictive model reliably predicted risk of type II diabetes in Asian populations
}

\author{
Calvin Woon-Loong Chin ${ }^{1}$, Elian Hui San Chia ${ }^{5}$, Stefan $\mathrm{Ma}^{2}$, Derrick Heng ${ }^{2}$, Maudrene $\operatorname{Tan}^{3}$, Jeanette Lee ${ }^{5}$, \\ E Shyong Tai ${ }^{4,5}$ and Agus Salim ${ }^{5^{*}}$
}

\begin{abstract}
Background: Identification of high-risk individuals is crucial for effective implementation of type 2 diabetes mellitus prevention programs. Several studies have shown that multivariable predictive functions perform as well as the 2-hour post-challenge glucose in identifying these high-risk individuals. The performance of these functions in Asian populations, where the rise in prevalence of type 2 diabetes mellitus is expected to be the greatest in the next several decades, is relatively unknown.

Methods: Using data from three Asian populations in Singapore, we compared the performance of three multivariate predictive models in terms of their discriminatory power and calibration quality: the San Antonio Health Study model, Atherosclerosis Risk in Communities model and the Framingham model.
\end{abstract}

Results: The San Antonio Health Study and Atherosclerosis Risk in Communities models had better discriminative powers than using only fasting plasma glucose or the 2-hour post-challenge glucose. However, the Framingham model did not perform significantly better than fasting glucose or the 2-hour post-challenge glucose. All published models suffered from poor calibration. After recalibration, the Atherosclerosis Risk in Communities model achieved good calibration, the San Antonio Health Study model showed a significant lack of fit in females and the Framingham model showed a significant lack of fit in both females and males.

Conclusions: We conclude that adoption of the ARIC model for Asian populations is feasible and highly recommended when local prospective data is unavailable.

\section{Background}

Type 2 diabetes mellitus (T2DM) and its associated complications have imposed a massive burden on public health care systems. Intensive lifestyle modification has been shown to effectively prevent or delay the development of T2DM [1-3]. Although effective, these interventional programs do incur some health care costs. In the Diabetes Prevention Program in the United States, the societal cost of lifestyle intervention was $\$ 3,540$ more per individual than the placebo group over 3 years [4]. This cost would prevent one case of diabetes for every 6.9 persons treated with lifestyle modification programs over 3 years [1]. The number of persons that needs to be treated to prevent one case of T2DM-and thus the cost-effectiveness of the program-is highly dependent

\footnotetext{
* Correspondence: ephaguss@nus.edu.sg

${ }^{5}$ Saw Swee Hock School of Public Health, National University of Singapore,

MD3, 16 Medical Drive, Singapore 117597, Singapore

Full list of author information is available at the end of the article
}

on the absolute risk of developing T2DM in the population undergoing the intervention. Thus, some form of risk assessment, with intervention targeted at those at highest risk, is critical to ensure the cost-effectiveness of such programs.

To date, randomized clinical trials have been relying on the diagnosis of impaired glucose tolerance (IGT) to selectively identify a population at high risk of T2DM. This diagnosis requires the administration of an oral glucose tolerance test (OGTT), which is inconvenient to both the clinician administering the test and the patient, leading to some reluctance in using it. Recognizing this reluctance, the American Diabetic Association (ADA), in 1997, encouraged the use of fasting plasma glucose (FPG) rather than the OGTT for the diagnosis of T2DM [5]. Levels of FPG between 'normal' and 'diabetic' were classified as impaired fasting glucose (IFG), a category analogous to IGT. However, IFG may not identify all individuals with the same degree of risk as those with IGT [6,7].

\section{Biomed Central}


Several studies have shown that multivariable predictive functions using variables collected by medical history and fasting blood tests perform as well, if not better, than the 2-hour post-challenge glucose (2hPG) in identifying individuals at high or low risk of developing T2DM [8-10], thereby obviating the need for an OGTT. However, the broad utility of these functions is limited by the relative lack of studies that have assessed the ability of these functions to accurately predict the absolute risk of developing T2DM, which is important for the planning of interventional strategies for the prevention of T2DM. Ideally, a population-specific predictive function should be used for risk prediction $[8,10,11]$. However, suitable prospective data that would facilitate the development of these predictive functions is not always available, particularly in developing countries in the Asia-Pacific region, where the rise in the prevalence of T2DM is expected to be the greatest in the next several decades [12]. In this situation, applying predictive functions developed in an external population seems to be a reasonable alternative. However, differences in the average levels of risk factors and their effect sizes, length of follow-up and baseline incidence rates will likely complicate the evaluation. In particular, it is quite possible to underestimate or overestimate the actual risk due to these differences. Furthermore, there are several risk functions available $[8,13,14]$ and it is not clear which risk function would perform best when applied to the Asian populations.

In order to determine the viability of adopting an externally-developed predictive function for T2DM to the Asian populations, we evaluated the performance of three multivariate predictive functions for T2DM risk: the San Antonio Health Study (SAHS) model [8], the Atherosclerosis Risk in Communities Study (ARIC) model [13] and the Framingham Offspring Study (FRAM) model [14]. These three models were chosen because a recent study had shown that they had excellent discriminative and calibration qualities when applied to multiethnic cohorts in the United States [15]. However, in order to use these functions in Asian populations, further evaluation of these functions is needed. The functions were evaluated using data from Singaporean Chinese, Malay and Asian Indians who were followed up as part of the Singapore Prospective Study Program (SP2) in the 1992 National Health Survey (NHS-92).

\section{Methods \\ The 1992 Singapore National Health Survey (NHS-92) Cohort}

The 1992 Singapore National Health Survey (NHS-92) was a cross sectional study carried out between September and November 1992 to determine the risk factors for major non-communicable diseases in Singapore. The results and methodology have been previously reported
[16]. Systematic sampling from a national household database, followed by disproportionate stratified sampling by ethnic groups, was used to select the sample for the survey. The two minority ethnic groups, Malays and Asian Indians, were over-sampled to obtain an ethnic distribution of approximately $60 \%$ Chinese, $20 \%$ Malays and 20\% Asian Indians to ensure sufficient numbers for statistical analysis. A total of 4,915 individuals were invited to join the study. Out of this, 3,568 individuals (response rate $=72.6 \%$ ) agreed to enrol in the current study (Figure 1). Response rates were significantly different across ethnic groups, with the Chinese having a significantly higher response rate compared to other ethnic groups [16].

The individuals who agreed to participate in NHS-92 survey completed an interviewer-administered questionnaire and underwent a baseline physical examination, which included anthropometric measurements (including height, weight and waist circumference), blood pressure, and measurements of fasting plasma glucose and lipids. Height (to the nearest millimeter) was recorded in all subjects without shoes, and weight (in kilograms) was measured, with subjects in light clothing, using electronic weighing scales (SECA model 220). Waist (defined as the narrowest part of the body below the costal margin) and hip (defined as the widest part of the body below the waist) measurements were also taken; fasting blood samples including serum lipids (10 ml plain tubes) and glucose ( $2 \mathrm{ml}$ fluoride oxalate tubes) were taken from 3,566 out of 3,568 subjects after an overnight fast of 10 hours (two subjects failed or refused venesection, and were thus excluded). OGTT was given to 3,435 subjects who were not on oral hypoglycemic medications or insulin (a completion rate of 96.3\%). Plasma glucose and lipid measurements were performed on the same day as blood collection.

\section{Follow-up of NHS-92 participants}

At baseline, there were 156 subjects with a previous diagnosis of T2DM. A further 214 of the NHS-92 subjects were diagnosed with diabetes mellitus at baseline, which was defined as FPG $\geq 7.0 \mathrm{mmol} / \mathrm{L}$ or $2 \mathrm{hPG} \geq 11.1 \mathrm{mmol} / \mathrm{L}$. These subjects were excluded, leaving 3,196 individuals in the NHS-92 cohort. Between 2004 and 2007, we conducted a study to follow up these individuals. The follow-up study consisted of an interviewer-administered questionnaire and a health screening, which included physical examination and blood tests. Ethics approval was obtained from the two Institutional Review Boards (National University of Singapore and Singapore General Hospital) prior to commencement of the study. Informed consent was obtained from the participants. The participants who were deceased at the time of follow-up (as shown by data-linkage with the Registry of Births and Deaths) were excluded from the study. The participants, whose contact details were provided by 


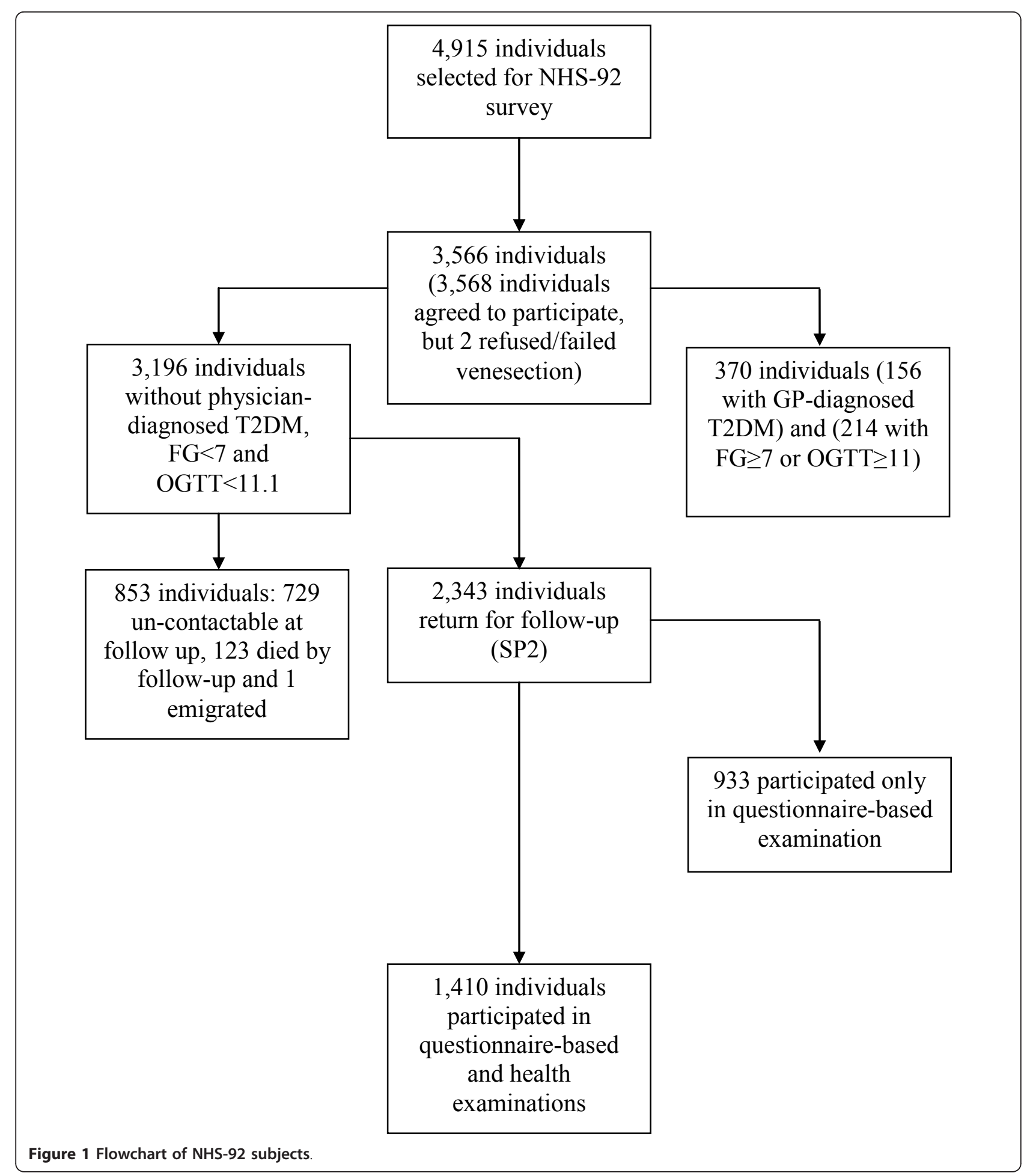

the Ministry of Home Affairs using their unique National Registration Identification Card number, were contacted first by mail, then by phone. Trained field interviewers conducted a home visit if there was no response or no available phone number. A minimum of three home visits at different times of the day (including a weekday and a weekend) were attempted before a participant was deemed unreachable.

Out of 3,196 individuals at baseline, 123 individuals had died, one emigrated and 729 individuals were not contactable at follow-up (Figure 1). The remaining 2,343 individuals were surveyed using interviewer-administered 
questionnaires to gather information on demographics and medical history during the home visits. Participants who returned were older, slightly more obese and less likely to be Chinese (Table 1). Those who did not return had lower baseline FPG and higher serum high density lipoprotein (HDL), and were less likely to have a family history of T2DM (Table 1). Subsequently, all the participants were invited to attend a health examination following a 10-hour overnight fast. A total of 1,410 individuals agreed to attend the health examination (Figure 1). Fasting blood specimens of these individuals were collected in fluoride oxalate tubes and sent in containers maintained at a temperature of $4^{\circ} \mathrm{C}$ for same-day analysis at the Referral Laboratory of the National University Hospital, Singapore, an American College of Pathologists-accredited laboratory. FPG was measured by enzymatic methods (ADVIA 2400, Bayer Diagnostics), with the blood collected in fluoride oxalate tubes. No OGTT was administered at follow-up. The outcome of interest was incident T2DM at follow-up, defined as having a FPG $\geq 7.0 \mathrm{mmol} / \mathrm{L}$ at follow-up or a physician diagnosis of T2DM.

\section{Statistical approaches Model development}

We excluded 370 individuals who had been previously diagnosed with T2DM by a physician or were diagnosed with T2DM (FPG $\geq 7.0 \mathrm{mmol} / \mathrm{L}$ or $2 \mathrm{hPG} \geq 11.1 \mathrm{mmol} /$ L) at baseline. A further 853 individuals who had died, emigrated or were unreachable at follow-up were also excluded. This left us with 2,343 participants who returned for follow-up, out of which 1,410 individuals attended the health examination (Figure 1) and therefore had their FPG measured at follow-up. However, nine individuals with FPG measurement at follow-up did not have complete baseline information needed to build and evaluate the predictive functions. Hence, the final number of subjects included for statistical analysis was 1401 .

For each published multivariate function, logistic regression was used to estimate the local version of the function. By local version of the function, we mean a model that used the same set of predictors as the corresponding published model, but whose coefficients were estimated using data from the NHS-92 cohort. In addition to the local version of the published multivariate models, two local models that used only FPG and 2hPG as predictors were estimated. In the SAHS and ARIC models, a dichotomous ethnicity variable was used as part of the predictive function. To enable application of these two models to the Singaporean population, individuals in the NHS-92 cohort were categorized into two categories, with Chinese ethnicity as the reference category, and non-Chinese ethnicity to include Malays and Asian Indians. The choice of Chinese as the reference category was motivated by the fact that prior research had found that the Chinese population in Singapore had lower incidence rates of T2DM than the Malays and Asian Indians. This choice is comparable to the choice in ARIC and SAHS models, where ethnic groups with lower incidence rates were chosen as the reference group for the studies. For the local version of the ARIC and SAHS models, we initially had two separate dummy variables for Malays and Asian Indians, but these two dummy variables were combined when the goodness of fit for model with one combined race variable is better than using two separate race variables.

For all multivariate models, between-population heterogeneity was investigated by examining the interaction terms between covariates in the model and ethnicity. Interaction terms found to be statistically significant using likelihood ratio test $(P<0.05)$ were subsequently added to the local models.

Since the local models were estimated using only 1,401 out of 2,343 subjects who returned for follow-up, we assessed the impact of excluding nine individuals with incomplete baseline information and 933 individuals with missing FPG measurements at follow-up. We did this by performing multiple imputation on the incomplete observations. For each of the 942 subjects with missing baseline information or FPG at follow-up, 20 imputed values are generated for the missing information using fully conditional specification (FCS) imputation $[17,18]$. The following baseline variables are used in the regression model for predicting FPG levels at follow-up (imputer model): age, gender, race, systolic blood pressure, body mass index (BMI), high density lipoprotein (HDL), low density lipoprotein (LDL), FPG at baseline, family history of T2DM, hip circumference, waist circumference, waist-hip ratio, creatinine levels, triglyceride and insulin resistance. To obtain the estimates under multiple imputation, logistic regression was fitted to each of the 20 imputed datasets separately. The multiple imputation estimates were obtained as the average estimates across the imputed datasets and the standard errors of the estimates were computed using Rubin's formula by taking into account both within and between imputation variance.

The probability that an individual developed T2DM during the follow-up period was estimated using the following formula: $p=1 /\left(1+e^{-y}\right)$, where $p$ is probability of incident diabetes $y$ is the logistic regression function.

\section{Model comparisons \\ Discrimination power}

The predictive functions were compared in terms of the discriminative power and calibration quality. To assess the discrimination power of a predictive function, we used the area under the receiver operating characteristic curves (AUC). The AUC represents an estimate of the 
Table 1 Baseline characteristics of participants without diabetes in the National Health Survey 1992

Baseline Characteristics National Health Survey 1992

\begin{tabular}{|c|c|c|c|c|c|c|}
\hline & \multicolumn{6}{|c|}{ Total number of participants without diabetes at baseline, $\mathrm{N}=3,196$} \\
\hline & \multirow{2}{*}{$\begin{array}{l}\text { A. } \\
\begin{array}{l}\text { Participants who did the survey and FPG during follow-up and } \\
\text { have complete baseline information } \\
n=1,401\end{array}\end{array}$} & \multirow{2}{*}{$\begin{array}{l}\text { B. } \\
\text { Participants who did only the } \\
\text { survey during follow-up } \\
n=942\end{array}$} & \multirow{2}{*}{$\begin{array}{l}\text { C. } \\
\text { Participants who did not } \\
\text { return for follow-up } \\
n=729^{*}\end{array}$} & \multicolumn{3}{|c|}{$P$ value } \\
\hline & & & & $\begin{array}{c}\text { A } \\
\text { vs. B }\end{array}$ & $\begin{array}{c}\text { A } \\
\text { vs. C }\end{array}$ & $\begin{array}{l}(A+B) \\
\text { vs. C }\end{array}$ \\
\hline Age, years & $36.14 \pm 10.84$ & $36.59 \pm 13.00$ & $34.01 \pm 12.32$ & 0.36 & $\begin{array}{c}< \\
0.001\end{array}$ & $<0.001$ \\
\hline Males, n (\%) & $669(47.8)$ & $450(47.6)$ & $333(45.7)$ & 0.93 & 0.36 & 0.34 \\
\hline Females, n (\%) & $732(52.2)$ & $496(52.4)$ & $396(54.3)$ & 0.93 & 0.36 & 0.34 \\
\hline Chinese, n (\%) & $924(66.0)$ & $592(62.6)$ & $517(70.9)$ & 0.09 & 0.02 & 0.002 \\
\hline Malay, n (\%) & $245(17.5)$ & $206(21.8)$ & $121(16.6)$ & 0.01 & 0.61 & 0.11 \\
\hline Asian Indian, n (\%) & $232(16.5)$ & $148(15.6)$ & $91(12.5)$ & 0.55 & 0.01 & 0.01 \\
\hline BMI (kg/m2) & $22.80 \pm 3.85$ & $22.92 \pm 4.11$ & $22.41 \pm 4.07$ & 0.47 & 0.03 & 0.009 \\
\hline FPG (mmol/L) & $5.35 \pm 0.44$ & $5.38 \pm 0.49$ & $5.27 \pm 0.43$ & 0.12 & $\begin{array}{c}<< \\
0.001\end{array}$ & $<0.001$ \\
\hline $\mathrm{HDL}(\mathrm{mmol} / \mathrm{L})$ & $1.26 \pm 0.31$ & $1.27 \pm 0.31$ & $1.31 \pm 0.31$ & 0.44 & $\begin{array}{c}< \\
0.001 \\
\end{array}$ & 0.002 \\
\hline $\mathrm{SBP}(\mathrm{mmHg})$ & $114.45 \pm 14.58$ & $117.15 \pm 17.30$ & $114.89 \pm 16.13$ & $\begin{array}{c}< \\
0.001\end{array}$ & 0.52 & 0.34 \\
\hline $\begin{array}{c}\text { Positive family history of } \\
\text { T2DM, } n(\%)\end{array}$ & $497(35.5)$ & $237(25.1)$ & $175(24.0)$ & $\begin{array}{c}<< \\
0.001\end{array}$ & $\begin{array}{c}<< \\
0.001\end{array}$ & $<0.001$ \\
\hline
\end{tabular}

* Excluded deceased individuals and individuals who moved out of the country 
probability that a model assigns a higher risk to those who develop T2DM during the follow-up period than to those who do not. When comparing the AUC of two different models, we used the method by DeLong et al. [19], with $P<0.05$ to indicate statistical significance.

We also used a more sensitive method called Net Reclassification Index (NRI) [20] for comparing the discrimination power of the published models. For NRI calculation, the predicted probabilities from each model were classified into $0-10 \%, 10.1-20 \%, 20.1-30 \%, 30.1-40 \%, 40.1-$ $50 \%$ and $>50 \%$ risk categories. A better reclassification for a case takes place whenever a case is placed at a higher risk category, while for non-cases, the opposite is true.

As we were interested in the applicability of the published model where local prospective data was not available, we compared the performance of the predictive models in the following manner: for each multivariate model, the discriminative ability of the published model was compared to the local model. The objective here was to assess the 'gap' between the published model and the best model developed from local data, had the data been available. The published models were also compared to the local models that used only 2hPG or FPG as a predictor. The objective of this last comparison was to assess the potential for using externally-developed predictive model as an alternative to the $2 \mathrm{hPG}$ or FPG for identifying high-risk individuals. Finally, comparisons between the published models were made to examine which published model had the best performance and thus could potentially be recommended for use in Asian populations.

\section{Calibration quality}

The Hosmer-Lemeshow goodness of fit test was used to assess the calibration quality of the various predictive models. For all models, the predicted and observed number of events in each quintile of estimated probability were calculated and the test statistic was compared to the $\chi^{2}$ statistic with 3 degrees of freedom. Values of the test statistic exceeding $11.5(P<0.01)$ implied a significant lack of calibration [21]. For the published models, we also performed the recalibration step (see Appendix A) to assess the extent to which the lack of calibration was caused simply by differences in baseline incidence rates and average risk factors between the two populations. To perform the recalibration, we needed to estimate from the local population the average values for each risk factor and the probability that a subject with the average risk factors survived the study period without being diagnosed with T2DM. The HosmerLemeshow statistics were computed both before and after recalibration to assess the effect of recalibration on the performance of the externally derived predictive function.
All statistical analyses were carried out using R 2.9.0 http://www.r-project.org and STATA version 10 (Stata Corporation, College Station, TX).

\section{Results}

For all local predictive models, no interactions between ethnicity and other covariates were found to be significant, so no interaction term was added to any of the local predictive models. local ARIC and SAHS models with one combined race variable for Malays and Asian Indians had better model fit than their counterparts with two separate race variables. For local SAHS models, the Akaike Information Criterion (AIC) for models with one and two race variables were 588.6 and 590.6 , respectively. For local ARIC models, the AIC were 587.9 and 589.8, respectively.

Table 2 shows comparisons of the coefficients of the three multivariate predictive functions that were estimated using the NHS-92 cohort with the published coefficients from the SAHS, ARIC and Framingham models.

For the ARIC model, the 95\% confidence intervals of the following risk factors did not include the published effect size from the original study: age, fasting FPG at baseline, waist circumference and triglyceride.

For the Framingham model, all locally-estimated effect size agreed well with the published ones, except for the effect of overweight, which was found to be significantly higher in our local model.

However, at this stage, we did not know to what extent the estimates from the local models had been influenced by the exclusion of 942 subjects without FPG measurement at follow-up or due to incomplete baseline information.

Table 3 shows the multiple imputation estimates for the three local models. Interestingly, after taking into account the dropout effects, the discrepancies between the local and published models were less startling. In particular, for the SAHS and ARIC models, the discrepancies in terms of FPG and measures of adiposity (BMI or waist circumference) were no longer statistically significant. There were, however, significantly smaller age and gender effect sizes when compared to the published models. With the Framingham model, we found a significantly larger effect of overweight, quite possibly because a Caucasian definition of overweight $(\mathrm{BMI} \geq 25)$ had been used instead of the WHO recommendation for Singapore, which used a cut-off of BMI $\geq 23$ to define overweight individuals [22].

\section{Discrimination power}

Table 4 compares AUC for the various predictive functions. Although the AUC for all three locally-estimated multivariate models were slightly higher than the 
Table 2 Comparison of published and locally-estimated multivariate predictive functions

\begin{tabular}{|c|c|c|c|c|c|c|}
\hline \multirow[b]{2}{*}{ Risk factor at baseline } & \multicolumn{2}{|l|}{ SAHS Model } & \multicolumn{2}{|l|}{ ARIC Model } & \multicolumn{2}{|c|}{ Framingham Model* } \\
\hline & locally-estimated $(95 \% \mathrm{Cl})$ & Published & $\begin{array}{c}\text { locally-estimated } \\
(95 \% \mathrm{CI})\end{array}$ & Published & locally-estimated $(95 \% \mathrm{Cl})$ & Published \\
\hline Age (years) & $-0.004(-0.026 ; 0.018)$ & 0.028 & $-0.023(-0.047 ; 0.000)$ & 0.0173 & NA & NA \\
\hline Gender & $0.098(-0.391 ; 0.588)$ & 0.661 & NA & NA & NA & NA \\
\hline Ethnicity & $0.646(0.186 ; 1.107)$ & 0.412 & $0.677(0.214 ; 1.140)$ & 0.4433 & NA & NA \\
\hline FPG $(\mathrm{mg} / \mathrm{dl})$ & $0.119(0.090 ; 0.148)$ & 0.079 & $0.120(0.090 ; 0.149)$ & 0.0880 & NA & NA \\
\hline IFG & NA & NA & NA & NA & $1.795(1.259 ; 2.331)$ & 1.98 \\
\hline $\mathrm{SBP}(\mathrm{mmHg})$ & $0.015(0.000 ; 0.030)$ & 0.018 & $0.015(0.000 ; 0.029)$ & 0.0111 & $0.843(0.372 ; 1.315)$ & 0.50 \\
\hline Triglyceride(mg) & NA & NA & $0.000(-0.001 ; 0.001)$ & 0.0027 & $0.695(0.232 ; 1.158)$ & 0.58 \\
\hline $\mathrm{HDL}(\mathrm{mg} / \mathrm{dl})$ & $-0.033(-0.058 ;-0.008)$ & -0.039 & $-0.027(-0.053 ;-0.001)$ & -0.0122 & $0.498(0.032 ; 0.965)$ & 0.94 \\
\hline BMI $\left(\mathrm{kg} / \mathrm{m}^{2}\right)$ & $0.154(0.097 ; 0.212)$ & 0.070 & NA & NA & NA & NA \\
\hline BMI (overweight) & NA & NA & NA & NA & $1.206(0.728 ; 1.683)$ & 0.30 \\
\hline BMI (obese) & NA & NA & NA & NA & $1.574(0.881 ; 2.267)$ & 0.92 \\
\hline Waist (cm) & NA & NA & $0.071(0.046 ; 0.096)$ & 0.0273 & & NA \\
\hline Height (cm) & NA & NA & $-0.044(-0.072 ;-0.016)$ & -0.0326 & & NA \\
\hline Family history of T2DM & $0.447(-0.008 ; 0.903)$ & 0.481 & $0.473(0.018 ; 0.928)$ & 0.4981 & $0.548(0.111 ; 0.986)$ & 0.57 \\
\hline
\end{tabular}

Bold and italicized fonts: Estimates from local models whose $95 \%$ confidence intervals do not overlap with the published estimates, i.e. we assumed that the published estimates were the true values in the hypothesis testing

${ }^{*}$ Categorical variables are used for the Framingham model with the following rule: fasting glucose level: $(1=100-125 \mathrm{mg} / \mathrm{dL}, 0 \leq 100 \mathrm{mg} / \mathrm{dL})$, overweight $(1=$ $\mathrm{BMI} \geq 25 \& \mathrm{BMI}<30,0=$ otherwise), obese ( $1=\mathrm{BMI} \geq 30,0=$ otherwise), low high density lipoprotein (HDL) $(1=\mathrm{HDL}<40 \mathrm{mg} / \mathrm{dL}$ in $\mathrm{men}$ or $<50 \mathrm{mg} / \mathrm{dL}$ in women, 0 = otherwise), high triglyceride level ( $1 \geq 150 \mathrm{mg} / \mathrm{dL}, 0=$ otherwise), elevated blood pressure (systolic blood pressure $\geq 130$ or diastolic blood pressure $\geq 85 \mathrm{~mm} \mathrm{Hg}, 0$ = otherwise)For the SAHS model, the 95\% confidence intervals of the coefficients estimated from the NHS-92 cohort included the published SAHS coefficient for all but four risk factors: age, gender, FPG at baseline and BMl; the coefficients for age and gender were greater for the published SAHS model than the locally-estimated coefficients. In contrast, the locally estimated coefficients for FPG and BMI were larger than the published coefficients.

corresponding statistic for their published counterpart, only in the case of SAHS model did this difference achieve statistical significance. All locally-estimated multivariate models achieved better discrimination power when compared to model that used FPG only (all $P<0.001$, Table 4), while the locally-estimated Framingham model is the only one that was not statistically better than model that used only 2hPG $(P=0.110)$.

Table 3 Comparisons of published and locally-estimated multivariate predictive functions after inclusion of 942 subjects with incomplete baseline and follow-up measurements using multiple imputations

\begin{tabular}{|c|c|c|c|c|c|c|}
\hline \multirow[b]{2}{*}{ Risk factor at baseline } & \multicolumn{2}{|l|}{ SAHS } & \multicolumn{2}{|l|}{ ARIC } & \multicolumn{2}{|c|}{ Framingham* } \\
\hline & $\begin{array}{l}\text { Locally-estimated } \\
\qquad(95 \% \mathrm{Cl})\end{array}$ & Published & $\begin{array}{l}\text { Locally-estimated } \\
\qquad(95 \% \mathrm{Cl})\end{array}$ & Published & $\begin{array}{l}\text { Locally-estimated } \\
\qquad(95 \% \mathrm{Cl})\end{array}$ & Published \\
\hline Age (years) & $-0.002(-0.018 ; 0.014)$ & 0.028 & $-0.014(-0.031 ; 0.003)$ & 0.0173 & NA & NA \\
\hline Gender & $0.182(-0.207 ; 0.570)$ & 0.661 & NA & NA & NA & NA \\
\hline Ethnicity & $0.371(0.024 ; 0.719)$ & 0.412 & $0.412(0.064 ; 0.759)$ & 0.4433 & NA & NA \\
\hline FPG (mg/dl) & $0.094(0.055 ; 0.134)$ & 0.079 & $0.080(0.056 ; 0.104)$ & 0.0880 & NA & NA \\
\hline IFG & NA & NA & NA & NA & $1.693(1.276 ; 2.110)$ & 1.98 \\
\hline $\mathrm{SBP}(\mathrm{mm} \mathrm{Hg})$ & $0.012(0.001 ; 0.023)$ & 0.018 & $0.011(0.000 ; 0.022)$ & 0.0111 & $0.584(0.215 ; 0.953)$ & 0.50 \\
\hline Triglyceride(mg/dl) & NA & NA & $0.000(-0.001 ; 0.001)$ & 0.0027 & $0.577(0.179 ; 0.976)$ & 0.58 \\
\hline $\mathrm{HDL}(\mathrm{mg} / \mathrm{dl})$ & $-0.021(-0.039 ;-0.004)$ & -0.039 & $-0.012(-0.029 ; 0.005)$ & -0.0122 & $0.303(-0.051 ; 0.656)$ & 0.94 \\
\hline BMI $\left(\mathrm{kg} / \mathrm{m}^{2}\right)$ & $0.080(0.057 ; 0.104)$ & 0.070 & NA & NA & NA & NA \\
\hline BMI (overweight) & NA & NA & NA & NA & $0.755(0.389 ; 1.12)$ & 0.30 \\
\hline BMI (obese) & NA & NA & NA & NA & $1.209(0.689 ; 1.729)$ & 0.92 \\
\hline Waist (cm) & NA & NA & $0.045(0.026 ; 0.063)$ & 0.0273 & NA & NA \\
\hline Height (cm) & NA & NA & $-0.030(-0.050 ;-0.011)$ & -0.0326 & NA & NA \\
\hline Family history of T2DM & $0.513(0.190 ; 0.837)$ & 0.481 & $0.541(0.215 ; 0.866)$ & 0.4981 & $0.625(0.300 ; 0.949)$ & 0.57 \\
\hline
\end{tabular}

Bold and italicized fonts: Estimates from local models whose $95 \%$ confidence intervals do not overlap with the published estimates, i.e., we assumed that the published estimates were the true values in the hypothesis testing)

${ }^{*}$ Categorical variables are used for the Framingham model with the following rule: fasting glucose level: $(1=100-125 \mathrm{mg} / \mathrm{dL}, 0 \leq 100 \mathrm{mg} / \mathrm{dL})$, overweight $(1=$ $\mathrm{BMI} \geq 25 \& \mathrm{BMI}<30,0=$ otherwise), obese ( $1=\mathrm{BMI} \geq 30,0=$ otherwise), low high density lipoprotein $(\mathrm{HDL})(1=\mathrm{HDL}<40 \mathrm{mg} / \mathrm{dL}$ in $\mathrm{men}$ or $<50 \mathrm{mg} / \mathrm{dL}$ in women, $0=$ otherwise), high triglyceride level ( $1 \geq 150 \mathrm{mg} / \mathrm{dL}, 0=$ otherwise), elevated blood pressure (systolic blood pressure $\geq 130$ or diastolic blood pressure $\geq 85 \mathrm{~mm} \mathrm{Hg}, 0=$ otherwise) 
Table 4 Comparisons of area under the Receiver Operating Characteristic curve (AUC) for various predictive models evaluated using NHS-92 Cohort

\begin{tabular}{|c|c|c|c|c|}
\hline Model 1 & $\begin{array}{c}\text { AUC } \\
(95 \% \text { C.I) }\end{array}$ & Model 2 & $\begin{array}{c}\text { AUC } \\
(95 \% \text { C.I) }\end{array}$ & $P$ value for comparisor \\
\hline SAHS-local & $0.857(0.821 ; 0.894)$ & SAHS-published & $0.839(0.803 ; 0.874)$ & 0.028 \\
\hline ARIC-local & $0.864(0.829 ; 0.899)$ & ARIC-published & $0.847(0.812 ; 0.883)$ & 0.065 \\
\hline FRAM-local & $0.826(0.784 ; 0.867)$ & FRAM-published & $0.805(0.762 ; 0.849)$ & 0.076 \\
\hline SAHS-published & $0.839(0.803 ; 0.874)$ & ARIC-published & $0.847(0.812 ; 0.883)$ & 0.230 \\
\hline SAHS-published & $0.839(0.803 ; 0.874)$ & FRAM-published & $0.805(0.762 ; 0.849)$ & 0.028 \\
\hline ARIC-published & $0.847(0.812 ; 0.883)$ & FRAM-published & $0.805(0.762 ; 0.849)$ & 0.007 \\
\hline \multirow[t]{3}{*}{ FPG-local } & $0.782(0.736 ; 0.828)$ & SAHS-local & $0.857(0.821 ; 0.894)$ & $<0.001$ \\
\hline & & ARIC-local & $0.864(0.829 ; 0.899)$ & $<0.001$ \\
\hline & & FRAM-local & $0.826(0.784 ; 0.867)$ & 0.047 \\
\hline \multirow[t]{3}{*}{ OGTT-local } & $0.778(0.727 ; 0.828)$ & SAHS-local & $0.857(0.821 ; 0.894)$ & 0.004 \\
\hline & & ARIC-local & $0.864(0.829 ; 0.899)$ & 0.002 \\
\hline & & FRAM-local & $0.826(0.784 ; 0.867)$ & 0.110 \\
\hline \multirow[t]{3}{*}{ FPG-local } & $0.782(0.736 ; 0.828)$ & SAHS-published & $0.839(0.803 ; 0.874)$ & $<0.001$ \\
\hline & & ARIC-published & $0.847(0.812 ; 0.883)$ & $<0.001$ \\
\hline & & FRAM-published & $0.805(0.762 ; 0.849)$ & 0.323 \\
\hline \multirow[t]{3}{*}{ OGTT-local } & $0.778(0.727 ; 0.828)$ & SAHS-published & $0.839(0.803 ; 0.874)$ & 0.021 \\
\hline & & ARIC-published & $0.847(0.812 ; 0.883)$ & 0.011 \\
\hline & & FRAM-published & $0.805(0.762 ; 0.849)$ & 0.371 \\
\hline
\end{tabular}

Out of the three published functions, the ARIC model had the highest discrimination power $(\mathrm{AUC}=0.847)$, followed by the SAHS model (AUC $=0.839$ ) and the Framingham model (AUC $=0.805$ ). The performance of the published SAHS and ARIC models were not statistically different $(P=0.230)$, but both models had significantly higher discrimination power than the Framingham model ( $P=0.028$ and 0.007 , respectively). More importantly, the published SAHS and ARIC models were statistically better at discriminating T2DM cases from noncases when compared to the local model that used FPG only $(P<0.001)$ or 2 hPG only $(P=0.021$ and 0.011 , respectively).

The NRI statistic revealed that overall, the published ARIC model was only marginally better than the published SAHS model (NRI $=0.127, P=0.060)$. When we looked at cases and non-cases separately, the ARIC model was not significantly better than the SAHS model in terms of reclassifying cases (Figure 2). Specifically, compared to the SAHS model, 32 cases were appropriately reclassified by the ARIC model at a cost of 21 cases being reclassified inappropriately $(\mathrm{NRI}=0.100, P=0.131)$. However, the

\begin{tabular}{|c|c|c|c|c|c|c|c|c|c|c|c|c|c|}
\hline & & & a) ca & ses & & & & & $(b)$ & $\mathrm{On}-\mathrm{Ca}$ & ses & & \\
\hline $\mathrm{M}_{1}$ & $0-10 \%$ & $10-20 \%$ & $20-30 \%$ & $30-40 \%$ & $40-50 \%$ & $>50 \%$ & $\mathrm{M}_{1} / /$ & $0-10 \%$ & $10-20 \%$ & $20-30 \%$ & $30-40 \%$ & $40-50 \%$ & $>50 \%$ \\
\hline $0-10 \%$ & 23 & 10 & 0 & 0 & 0 & 0 & $0-10 \%$ & 984 & 30 & 0 & 0 & 0 & 0 \\
\hline $10-20 \%$ & 0 & 7 & 11 & 6 & 1 & 0 & $10-20 \%$ & 62 & 91 & 19 & 4 & 0 & 2 \\
\hline $20-30 \%$ & 1 & 4 & 8 & 7 & 1 & 0 & $20-30 \%$ & 1 & 20 & 15 & 10 & 1 & 1 \\
\hline $30-40 \%$ & 0 & 0 & 2 & 5 & 4 & 0 & $30-40 \%$ & 0 & 5 & 13 & 7 & 2 & 5 \\
\hline $40-50 \%$ & 0 & 0 & 1 & 2 & 1 & 3 & $40-50 \%$ & 0 & 0 & 6 & 1 & 3 & 2 \\
\hline$>50 \%$ & 0 & 0 & 1 & 0 & 3 & 8 & $>50 \%$ & 0 & 0 & 0 & 0 & 2 & 6 \\
\hline
\end{tabular}

Figure 2 Comparisons of risk classifications for subjects in NHS-92 cohort using recalibrated ARIC $\left(M_{1}\right)$ and SAHS $\left(M_{2}\right)$ models. 
Table 5 Calibration quality of various predictive models evaluated using NHS-92 Cohort $(N=1,401)$

\begin{tabular}{lcc}
\hline Model & HL-statistic & Predicted incidence (\%) \\
\hline SAHS-local & 3.52 & 7.8 \\
ARIC-local & 2.28 & 7.8 \\
FRAM-local & 1.45 & 7.8 \\
FPG-local & 3.08 & 7.8 \\
OGTT-local & 3.90 & 7.8 \\
SAHS-published & 48.33 & 13.5 \\
ARIC-published & 14.71 & 9.8 \\
FRAM-published & 264.89 & 2.0 \\
Recalibrated SAHS-published & 23.19 & 11.2 \\
Recalibrated ARIC-published & 10.31 & 8.5 \\
Recalibrated FRAM-published & 103.15 & 16.9 \\
\hline
\end{tabular}

ARIC model was better at reclassifying non-cases. In total, 110 non-cases were appropriately reclassified using the ARIC model, at a cost of 76 non-cases being reclassified inappropriately $(\mathrm{NRI}=0.026, P=0.013)$.

\section{Calibration}

The calibration inspections revealed that the local models showed good calibration properties (Table 5). In particular, the H-L statistics for local models are all less than 11.5 and the predicted incidence rates under all local models agree well with observed incidence rates over the 13-year period in the NHS-92 cohort, which was 7.8\%. However, the three published models showed poor calibration, with the Framingham model being the worst. In particular, the SAHS and ARIC published models overestimated the incidence rates, while the Framingham model underestimated the incidence rates. Specifically, the estimated incidence rates from the SAHS and ARIC models were $13.5 \%$ and $9.8 \%$, respectively. Meanwhile, estimated incidence rates from the Framingham model is $2.0 \%$.

Recalibration improved the calibration quality of ARIC model (Figure 3), but the same cannot be said for the Framingham model. The recalibration procedure seemed to work reasonably well for the SAHS model for subjects in the lowest three quintiles (Figure 3); however, the SAHS model still overestimated the number of cases in the two highest quintiles even after recalibration. The poor performance of the Framingham model could be due to the fact that the Framingham cohort used to derive the model consists almost exclusively of one race while the Singapore population consists of three races with Chinese being different from Malays and Indians. To investigate this possibility, we performed local fitting and recalibration of the three published models separately in the Chinese and non-Chinese populations, with the race terms removed from the ARIC and SAHS models. In the Chinese population, the H-L statistic for locally-fitted ARIC, Framingham and SAHS models is $4.22,3.43$ and 1.38 respectively, indicating good calibration properties. However, only recalibrated ARIC and SAHS models show acceptable calibration quality with $\mathrm{H}$-L statistic of 7.31 and 2.23 respectively. The recalibrated Framingham model still shows poor calibration quality $(\mathrm{H}-\mathrm{L}$ statistic $=26.12)$. Among non-Chinese population the story is very similar. The H-L statistic for locally-fitted ARIC, Framingham and SAHS models is 1.53, 2.74 and 3.34 respectively. Among the recalibrated published models, only ARIC shows acceptable calibration quality with $\mathrm{H}-\mathrm{L}$ statistic of 6.87 . The recalibrated Framingham and SAHS have poor calibration quality with H-L statistic of 19.29 and 140.17, respectively. Thus,

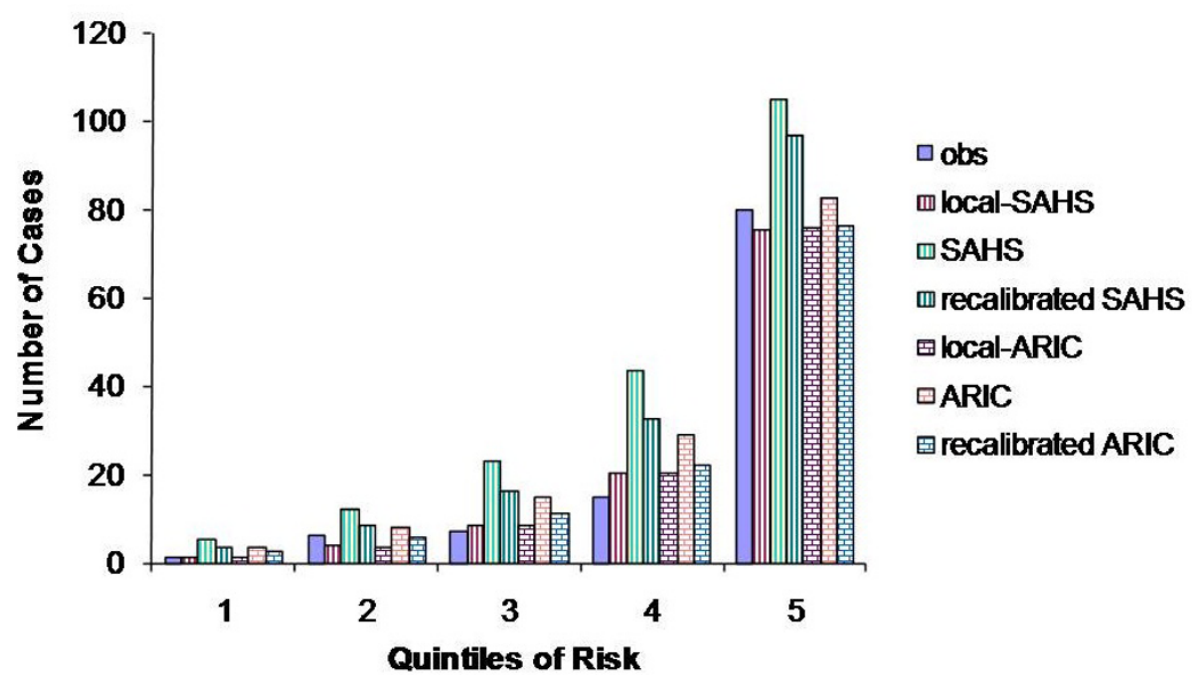

Figure 3 Observed and predicted number of cases in the different risk quintiles 
the poorer performance of the Framingham model is unlikely only due to differences in race effects between the Framingham cohort and Singapore population. It is more likely that differences in the effect sizes of some of the risk factors also contribute to the poor performance.

\section{Discussion}

Studies in the United States [8,9] and Asia [10] have shown that a multivariable predictive model that incorporated clinical risk factors and fasting blood tests performed better than $2 \mathrm{hPG}$ in predicting the risk of T2DM and could replace the OGTT in identifying individuals at increased risk of T2DM that would be suitable candidates for a diabetes prevention program. Our study confirms these findings.

We also found that externally-developed predictive models showed good discriminative power, even when applied to Asian ethnic groups. However, we noted that this did not apply equally to all predictive models. In our population, the discriminatory power of the published Framingham model [14] was not statistically better than risk prediction using FPG or 2hPG. Using a multiethnic US cohort, Mann et al. [15] also found that the performance of the Framingham model was significantly worse than ARIC and SAHS models. This may relate to the use of categorical variables in the Framingham model, compared to the use of continuous variables in the other models. Using NRI statistic, we also found that the published ARIC model was significantly better than the SAHS model in classifying subjects with low risk of developing T2DM. This was in contrast to the findings of Mann et al., who found the SAHS and ARIC models to be equivalent in terms of discriminative power. The reason that recalibration did not work very well for the SAHS model here was because the published model included gender effect, while, in our local population, we did not find differences between gender. The fact that discrepancy in gender effect was causing unsatisfactory recalibration performance was more apparent when we evaluated the calibration quality separately for females and males (Table 6). The recalibration worked well for the SAHS model among males where gender effects did

Table 6 Hosmer-Lemeshow (HL) statistics for various published predictive models evaluated using Female and Male subsets of NHS-92 cohort

\begin{tabular}{lcc}
\hline Model & Female $(\mathbf{N}=\mathbf{7 3 2})$ & Male $(\mathbf{N}=\mathbf{6 6 9})$ \\
\hline SAHS-published & 37.31 & 16.25 \\
ARIC-published & 7.41 & 14.17 \\
FRAM-published & 112.90 & 157.66 \\
Recalibrated SAHS-published & 23.06 & 7.11 \\
Recalibrated ARIC-published & 5.43 & 12.25 \\
Recalibrated FRAM-published & 53.35 & 51.22 \\
\hline
\end{tabular}

not exist (i.e. male being the reference category in the model), while recalibration did not work well for females.

The validity of externally-developed predictive models depends on the homogeneity of effect size, risk factors distribution and length of follow-up between the two populations. Differences in terms of the last two factors can be remedied using recalibration. Heterogeneity of effect sizes, however, is harder to remedy, and, when it exists, it tends to downgrade the performance of the externally-developed functions. In our case, we initially found a greater effect of baseline FPG and obesity (whether assessed using BMI or waist circumference) in our Asian cohort when compared to the US cohorts that were used to develop the various predictive functions. Other authors have previously reported findings that BMI underestimated the degree of adiposity in Asians as compared to Caucasian populations [23,24]. While we cannot exclude this possibility, our data suggests that these discrepancies may also be caused, to some extent, by the failure of our initial analyses to include those subjects without complete baseline data or FPG at follow-up. After the inclusion of these subjects via multiple imputation, the differences in the parameter estimates were no longer statistically significant. This highlights the importance of assessing dropout effects and avoiding the sole use of subjects with complete measurements when developing and comparing predictive functions. This is because subjects excluded from complete-case analysis often have very different characteristics from those included. Using multiple imputation is a reasonable approach for including individuals with incomplete data. However, multiple imputation does implicitly assume that the probability of individuals having incomplete data does not depend on the missing variable itself (in this case, FPG at follow-up), but, rather, it depends on a set of other variables that are observed. This mechanism of 'missingness' is called missing at random (MAR). The assumption is not testable, but it can be made more plausible by using a more inclusive strategy in which more variables are included in the imputer model for prediction of missing values. In our analysis, we used this strategy by including as many baseline variables that we thought may be good predictors of FPG at follow-up as possible. This strategy seems to work reasonably well, since the coefficients in the local models were more comparable to the published estimates, after multiple imputation. However, given that the amount of imputed data is substantial, we can never completely rule out the possibility of remaining bias caused by differences in the unobserved/unmeasured characteristics that are also associated with FPG at follow-up.

The published ARIC and SAHS models included binary 'race' variables in their models. The 'race' coefficient likely represents unique contributions from genetics and 
socio-economic factors in the external populations used to build the models, and, generally speaking, its validity needs to be examined carefully when it is applied to completely different populations. The assumption of having exactly two race groups in the models is also potentially problematic when the models are to be applied into different populations. Even when the external population has exactly two major race groups, without local fitting of the models, it is not necessarily obvious which group should be regarded as the lower risk group. If more than two race groups (such as in this manuscript), without local fitting it is not necessarily clear which groups to merge to get down to exactly two groups. We propose that when sufficient local data are available the coefficients for 'race' terms need to be re-estimated using local data. This will avoid assumptions that there are exactly two race groups in the local population and the magnitudes of the race terms and the difference between the races are similar in this population as in the populations used to derive the ARIC and SAHS models. When there are not enough local data, we suggest a two-step approach for risk prediction is used: at the first step, the risks prediction is done by removing the race terms in the models, followed by the second step where the predicted risks are recalibrated within each race (ethnic group). By removing the race terms the relative ordering of risks should be reasonable but the absolute risks are unlikely to be predicted accurately. The recalibration step is needed to ensure that the discrepancy in the absolute risks due to differences in race coefficients is minimized.

The use of 'age' coefficient should also be considered carefully when the age distribution in the population to which the models were to be applied is very different from the population in which the model was built. In our case, the average age of our subjects is significantly younger than the ARIC and SAHS cohorts. If there is a birth cohort effect or nonlinear age effect, this may explain why we failed to discover significant age effects in our local models. Indeed, when sufficient local data is available, performing recalibration for different age groups separately in a similar manner to the ethnic-specific recalibration above is arguably better than blindly extrapolating the age effects.

In addition, we also would like to draw attention to the heterogeneity in the T2DM definition employed by the different models. Ideally, T2DM is defined based on outcomes of both FPG and OGTT. However, due to lack of measurements, only the SAHS study used this definition consistently to derive its model. The Framingham model used FPG/OGTT to define T2DM at baseline; however, it relied only on FPG to identify incident T2DM at follow-up, while the ARIC model failed to incorporate OGTT when identifying T2DM cases at baseline. In our dataset, we used FPG/OGTT at baseline, but only FPG measurements were available to define incident T2DM at follow-up. While investigations using our FPG and OGTT data at baseline showed that the misclassification rates were not statistically related to ethnicity or gender (data not shown), the possibility of differential misclassification in which subjects with certain profiles is more likely than others to be diagnosed with T2DM using OGTT only, is real. Differential misclassification will likely affect the recalibration performance and thus potentially make prediction of absolute risk less reliable. However, we believe that predictive risk would still work adequately well for the purpose of classifying individuals into high-risk and low-risk groups.

\section{Conclusions}

In summary, we have confirmed that multivariate predictive functions based on clinical and biochemical measurements made in the fasting state outperform an OGTT for predicting future T2DM. In addition, we have shown that the effects of risk factors on the risk of incident T2DM are broadly similar in Asians as they are in other ethnic groups. This means that in populations where data from relatively large and well-documented prospective cohort needed for developing local predictive function is not available, the use of a predictive function derived in another population is feasible, and, arguably preferable, to using FPG or OGTT alone. The only requirement for the function to be applicable in another population would be the availability of a small, pilot cohort in the order of several hundred subjects, from which survival rates and average risk factors can be estimated and used for recalibration. Our data further suggests that if one were to choose from the three predictive functions tested, the ARIC predictive function should be the choice.

\section{Appendix}

\section{Appendix A: Recalibration Method}

Several authors e.g. $[25,26]$ have suggested the use of the following formula in order to recalibrate predicted probability computed using models developed in an external population,

$$
\mathrm{p}(\underline{\mathrm{x}})=1-\mathrm{S}(\underline{\mathrm{m}}) \exp \left((\mathrm{x}-\underline{\mathrm{m}})^{\prime} \underline{\beta}\right)
$$

where $\mathrm{p}(\underline{\mathbf{x}})$ is the recalibrated probability of an event for subjects with risk factors $\underline{\mathbf{x}}, \mathrm{S}(\underline{\mathbf{m}})$ is the survival rates for subjects with average risk factors $\underline{m}$ in the local population (in our case, the NHS-92 cohort) and $\underline{\beta}$ the vector of regression coefficients estimated from the external population. This recalibration formula can be expected to work satisfactorily when there are no or 
little differences between the risk factors and their magnitude in the external and local populations.

The recalibration formula was originally suggested to recalibrate Framingham CHD risk [25], where $\boldsymbol{\beta}$ is estimated using Cox proportional hazard model; however, for interval-censored data with relatively low incidence rates, $\underline{\beta}$ from logistic regression can also be used. The justification for this is because for interval-censored data, Cox proportional hazard model is the same as specifying complementary log-log regression model, which, in turn, is very similar to the logistic regression model when incidence rates are low.

\section{Acknowledgements}

This study was funded by National Medical Research Council (NMRC), Singapore. All authors declare no conflict of interests.

This research was supported Singapore Biomedical Research Council Grant No 03/1/27/18/216 and Singapore National Medical Research Council Grant No. $1261 / 2010$

\section{Author details}

'Department of Cardiology, National Heart Centre, Singapore, Singapore. ${ }^{2}$ Epidemiology and Disease Control Division, Ministry of Health, Singapore, Singapore. ${ }^{3}$ Office of Clinical Research, SingHealth, Singapore, Singapore. ${ }^{4}$ Department of Medicine, Yong Loo Lin School of Medicine, National University of Singapore, Singapore, Singapore. ${ }^{5}$ Saw Swee Hock School of Public Health, National University of Singapore, MD3, 16 Medical Drive, Singapore 117597, Singapore.

\section{Authors' contributions}

CC wrote the original version of manuscript and contributed to statistical analyses. SM and DH contributed to supervising the implementation of the National Health Survey (NHS) and the subsequent SP2 study. EST conceived the SP2 study and contributed to the Results and Discussion sections. $J L$ conceived the SP2 study and contributed to the Discussion section. MT contributed to the statistical analyses and maintained the quality of the survey database. EHSC contributed to the statistical analyses and wrote part of the Method section. AS directed and supervised the overall quality of statistical analyses and contributed to all parts of the manuscripts. All authors have read and approved the submitted manuscript.

\section{Competing interests}

The authors declare that they have no competing interests.

Received: 18 November 2010 Accepted: 13 April 2012

Published: 13 April 2012

\section{References}

1. Knowler WC, Barrett-Connor E, Fowler SE, Hamman RF, Lachin JM, Walker EA, Nathan DM, Diabetes Prevention Program Research Group: Reduction in the incidence of type 2 diabetes with lifestyle intervention or metformin. N Engl J Med 2002, 346:393-403.

2. Pan XR, Li GW, Hu YH, Wang JX, Yang WY, An ZX, Hu ZX, Lin J, Xiao JZ, Cao $H B$, Liu $P A$, Jiang $X G$, Jiang $Y Y$, Wang JP, Zheng $H$, Zhang $H$, Bennett PH, Howard BV: Effects of diet and exercise in preventing NIDDM in people with impaired glucose tolerance. The Da Qing IGT and Diabetes Study. Diabetes Care 1997, 20:537-544.

3. Tuomilehto J, Lindstrom J, Eriksson JG, Valle TT, Hämäläinen $H_{\text {, llanne- }}$ Parikka P, Keinänen-Kiukaanniemi S, Laakso M, Louheranta A, Rastas M, Salminen V, Uusitupa M, Finnish Diabetes Prevention Study Group: Prevention of type 2 diabetes by changes in lifestyle among subjects with impaired glucose tolerance. N Engl J Med 2001, 344:1343-1350.

4. Hernan WH, Brandle M, Zhang P, Williamson DF, Matulik MJ, Ratner RE, Lachin JM, Engelgau MM, Diabetes Prevention Program Research Group:
Costs associated with the primary prevention of type 2 diabetes mellitus in the diabetes prevention program. Diabetes Care 2003, 26:36-47.

5. Expert committee on the diagnostic and classification of diabetes mellitus: Report of the expert committee on the diagnosis and classification of diabetes mellitus. Diabetes Care 1997, 20:S5-S19.

6. Tai ES, Goh SY, Lee JJ, Wong MS, Heng D, Hughes K, Chew SK, Cutter J, Chew W, Gu K, Chia KS, Tan CE: Lowering the criterion for impaired fasting glucose: impact on disease prevalence and associated risk of diabetes and ischemic heart disease. Diabetes Care 2004, 27:1728-1734.

7. Vaccaro O, Ruffa G, Imperatore G, lovino V, Rivellese AA, Riccardi G: Risk of diabetes in the new diagnostic category of impaired fasting glucose: a prospective analysis. Diabetes Care 1999, 22:1490-1493.

8. Stern MP, Williams K, Haffner SM: Identification of persons at high risk for type 2 diabetes mellitus: do we need the oral glucose tolerance test? Ann Intern Med 2002, 136:575-581.

9. McNeely MJ, Boyko EJ, Leonetti DL, Kahn SE, Fujimoto WY: Comparison of a clinical model, the oral glucose tolerance test, and fasting glucose for prediction of type 2 diabetes risk in Japanese Americans. Diabetes Care 2003, 26:758-763.

10. Aekplakorn W, Bunnag P, Woodward M, Sritara P, Cheepudomwit S, Yamwong S, Yipintsoi T, Rajatanavin R: A risk score for predicting incident diabetes in the Thai population. Diabetes Care 2006, 29:1872-1877.

11. Chen L, Magliano DJ, Balkau B, Colagiuri S, Zimmet PZ, Tonkin AM, Mitchell P, Phillips PJ, Shaw JE: AUSDRISK: an Australian Type 2 Diabetes Risk Assessment Tool based on demographic, lifestyle and simple anthropometric measures. Med J Aust 2010, 192:197-202.

12. Haslam DW, James WP: Obesity. Lancet 2005, 366:1197-1209.

13. Schmidt MI, Duncan BB, Bang H, Pankow JS, Ballantyne CM, Golden SH, Folsom AR, Chambless LE, Atherosclerosis Risk in Communities Investigators: Identifying individuals at high risk for diabetes: the Atherosclerosis risk in communities study. Diabetes Care 2005, 28:2013-2018.

14. Wilson PW, Meigs JB, Sullivan L, Fox CS, Nathan DM, D'Agostino RB Sr: Prediction of incident diabetes mellitus in middle-aged adults: the Framingham Offspring Study. Arch Intern Med 2007, 167:1068-1074.

15. Mann DM, Bertoni BG, Shimbo D, Carnethon MR, Chen H, Jenny NS, Muntner P: Comparative validity of 3 diabetes mellitus risk prediction scoring models in a multiethnic US Cohort: the Multi-Ethnic Study of Atherosclerosis. Am J Epidemiol 2010, 171:980-988.

16. Tan CE, Emmanuel SC, Tan BY, Jacob E: Prevalence of diabetes and ethnic differences in cardiovascular risk factors. The 1992 Singapore National Health Survey. Diabetes Care 1999, 22:241-247.

17. van Buuren S, Boshuizen HC, Knook DL: Multiple imputation of missing blood pressure covariates in survival analysis. Stat Med 1999, 18:681-694.

18. Lee KJ, Carlin JB: Multiple imputation for missing data: fully conditional specification versus multivariate normal imputation. Am J Epidemiol 2010, 171:624-632.

19. DeLong ER, DeLong DM, Clarke-Pearson DL: Comparing the areas under two or more correlated receiver operating characteristic curves: a nonparametric approach. Biometrics 1988, 44:837-845.

20. Pencina MJ, D'Agostino RB Sr, D'Agostino RB Jr, Vasan RS: Evaluating the added predictive ability of a new marker: from area under the ROC curve to reclassification and beyond. Stat Med 2008, 27:157-172.

21. D'Agostino RB Sr, Grundy S, Sullivan LM, Wilson P, CHD Risk Prediction Group: Validation of the Framingham coronary heart disease prediction scores: results of a multiple ethnic groups investigation. JAMA 2001, 286:180-187.

22. Revision of Body Mass Index (BMI) cut off in Singapore 2005. [http:// www.hpb.gov.sg/hpb/default.asp?pg_id=1769].

23. Deurenberg $P$, Yap $M$, van Staveren WA: Body mass index and percent body fat: a meta analysis among different ethnic groups. Int J Obes Relat Metab Disord 1998, 22:1164-1171.

24. Deurenberg-Yap $M$, Chew SK, Deurenberg P: Elevated body fat percentage and cardiovascular risks at low body mass index levels among Singaporean Chinese, Malays and Indians. Obes Rev 2002, 3:209-215.

25. Liu J, Hong Y, D'Agostino RB Sr, Wu Z, Wang W, Sun J, Wilson PW, Kannel WB, Zhao D: Prediction value for the Chinese Population of the Framingham CHD risk assessment tool compared with the Chinese Multi-Provincial Cohort Study. JAMA 2004, 291:2591-2599. 
26. Marrugat J, D'Agostino R, Sullivan L, Elosua R, Wilson P, Ordovas J,

Solanas P, Cordón F, Ramos R, Sala J, Masiá R, Kannel WB: An adaptation of the Framingham coronary heart disease risk function to European

Mediterranean areas. J Epidemiol Community Health 2003, 57:634-638.

\section{Pre-publication history}

The pre-publication history for this paper can be accessed here:

http://www.biomedcentral.com/1471-2288/12/48/prepub

doi:10.1186/1471-2288-12-48

Cite this article as: Chin et al:: The ARIC predictive model reliably

predicted risk of type II diabetes in Asian populations. BMC Medical

Research Methodology 2012 12:48.

Submit your next manuscript to BioMed Central and take full advantage of:

- Convenient online submission

- Thorough peer review

- No space constraints or color figure charges

- Immediate publication on acceptance

- Inclusion in PubMed, CAS, Scopus and Google Scholar

- Research which is freely available for redistribution

Submit your manuscript at www.biomedcentral.com/submit 